\title{
南雄盆地白严纪-古近纪(K-Pg)界线位置探讨 来自火山活动及古气候演化的证据
}

赵梦婷 ${ }^{1}$,马明明 ${ }^{2,3 *}$, 何梅 ${ }^{1}$, 邱显丹 ${ }^{1}$, 刘秀铭 ${ }^{2,3,4}$

1. 福建师范大学地理科学学院, 福州 350007 ;

2. 福建师范大学湿润亚热带生态-地理过程教育部重点实验室, 福州 350007;

3. 福建师范大学湿润亚热带山地生态国家重点实验室培育基地, 福州 350007;

4. Department of Environment and Geography, Macquarie University, Sydney NSW 2109, Australia

* 通讯作者, E-mail: mamingming159@163.com

收稿日期: 2020-10-19; 收修改稿日期: 2021-01-09; 接受日期: 2021-02-02; 网络版发表日期: 2021-03-03

国家自然科学基金项目(批准号：41602185和41772180)、国际地球科学计划项目(编号：IGCP 679)和福建师范大学创新团队项目(编号: IRTL1705)资助

摘要陆相地层中 K-Pg位置的确定对白严纪末期陆地生态系统演化过程(尤其是非鸟类恐龙灭绝)的研究意义重 大，目前世界范围内的陆相K-Pg研究集中在中高纬度地区，如北美及中国的东北地区. 位于低纬度地区的南雄盆 地虽然也开展了大量的工作，成为中国陆相白严系-古近系地层划分对比的标准，但是不同的研究者从不同的角 度提出了 4 条可能的界线, 因此关于其具体的位置仍有待进一步确定. 本文对4条界线前后的样品进行了总录( $\mathrm{Hg}$ ) 含量、环境磁学、地球化学等参数的测定, 并与全球记录进行了对比. 结果显示: (1) 总 $\mathrm{Hg}$ 含量在浈水组上段和上 湖组坪岭段明显升高, 且表现出剧烈的峰谷波动, 结合已有的年代结果, 可以将 $\mathrm{Hg}$ 的异常高值归因为德干高原火 山喷发，界线1位于 $\mathrm{Hg}$ 异常区间的中部，也是火山喷发的中期，符合全球 $\mathrm{K}-\mathrm{Pg}$ 界线与火山喷发的时间关系; (2) 重 建的古气候演化曲线揭示出盆地红色沉积物记录了马斯特里赫特期末期的热事件( 66.2Ma, LMWE), 从4条界线 与该事件的联系上看, 也只有界线 1 最接近南雄盆地真实的 $\mathrm{K}-\mathrm{Pg}$ 界线.

关键词 南雄盆地, $\mathrm{K}-\mathrm{Pg}$ 界线, 总 $\mathrm{Hg}$ 含量, 马斯特里赫特期末期热事件

\section{1 引言}

白严纪-古近纪界线 $(\mathrm{K}-\mathrm{Pg})$ 前后伴随着德干高原 的喷发以及小行星的撞击，全球生物发生了一次显著 的大灭绝事件(Schulte等, 2010; Renne等, 2013), 最有 名的便是非鸟类恐龙的灭绝，这使得 $\mathrm{K}-\mathrm{Pg}$ 附近的古气
候、古生物研究成为国际热点问题. 目前全球K-Pg界 线层型剖面(GSSP)位于突尼斯El Kef剖面(Molina等, 2006), 为典型的海相沉积, 该剖面的确立无法解决陆 相领域的很多问题, 如非鸟类恐龙的灭绝及哺乳动物 的复苏, 因此有必要建立“陆相层型”(李伟同等, 2010). 从世界范围内来看, 陆相沉积中确定的 K-Pg界线大多

\footnotetext{
中文引用格式: 赵梦婷, 马明明, 何梅, 邱显丹, 刘秀铭. 2021. 南雄盆地白严纪-古近纪(K-Pg)界线位置探讨一一来自火山活动及古气候演化的证据. 中国科学: 地球科学, 51(5): 741-752, doi: 10.1360/SSTe-2020-0287

英文引用格式: Zhao M, Ma M, He M, Qiu Y, Liu X. 2021. Evaluation of the four potential Cretaceous-Paleogene (K-Pg) boundaries in the Nanxiong Basin based on evidences from volcanic activity and paleoclimatic evolution. Science China Earth Sciences, 64(4): 631-641, https://doi.org/10.1007/s11430-0209736-0
} 
位于北美地区，中国境内分布着众多白严纪-古近纪陆 相沉积盆地( $\mathrm{Li}$ 等, 2013), 研究程度比较高的有松辽盆 地(Wu等, 2009; Wan等, 2013; Wang等, 2013; Wang, 2013)、嘉荫盆地(孙革，2014)、南雄盆地(赵资奎等, 2009; 张显球等, 2013; 王尹等, 2015; Zhao等, 2002; Clyde等, 2010; Li等, 2010)等, 为陆相K-Pg界线及古气 候演变研究提供了材料. 研究者在中国不同地区提出 了多条K-Pg标准剖面, 如松辽盆地(李莎等, 2013; Wan 等, 2013)、嘉荫盆地(孙革, 2014)、南雄盆地(童永生 等, 2002, 2013; 凌秋贤等, 2005; 张显球和李罡, 2015; 赵资奎等, 2017; Erben等, 1995; Clyde等, 2010)、江汉 盆地(李伟同等，2014)、豫西栾川-潭头盆地(江小均 等, 2011)等, 遗憾的是各剖面中K-Pg界线的准确位置 仍悬而未决(席党鹏等, 2019). 一是没有发现类似于北 美地区的典型的孢粉组合变化(刘耕武等, 2009), 二是 各盆地中利用不同指标建立的 K-Pg界线位置大相径 庭(如南雄盆地). 因此有必要继续开展中国陆相沉积 的K-Pg界线研究.

位于中国东南地区的南雄盆地(图1)完整地保存 着上白严统-早古新统的红色、紫红色泥岩-砂岩, 出 露良好, 盆地内的大塘剖面是中国非海相白严系-古近 系地层划分对比的标准, 也是中国白严系-古近系地 层、古生物、非鸟类恐龙绝灭、 K-Pg界线研究最精 细的热点地区(张显球, 1984; 张显球等, 2013; 赵资奎 等, 2017; Erben等, 1995). 目前关于大塘剖面K-Pg位置 有4种观点(图1d)：1)位于上湖组底部灰黄色底砾岩中 (相当于赵资奎等(1991) CGY-CGD剖面的CGD162.5m 处; 张显球等(2006)大塘剖面的43/42层之间), 其依据 是非鸟类恐龙的灭绝、哺乳动物的出现、古地磁以及 $\delta^{13} \mathrm{C}$ 的负漂移(童永生等, 2002, 2013; Clyde等, 2010); 2)位于上湖组坪岭段上部(相当于赵资奎等(1991) CGY-CGD剖面的CGD231m处; 张显球等(2006)大塘剖 面的49/48层之间), 依据是大型哺乳类阶齿兽化石的 出现和非海相腹足类化石组合(余汶等, 1990; 张显球 和凌秋贤, 2004; 凌秋贤等, 2005); 3)位于上湖组坪岭 段与下惠段界线处(相当于赵资奎等(1991) CGY-CGD 剖面的CGD250m处; 张显球等(2006)大塘剖面的53/52 层之间), 依据是介形虫化石组合特征(张显球, 1984, 1992)以及综合的古生物群面貌(张显球和李罡, 2015); 4)位于浈水组上段下部约 $21 \mathrm{~m}$ 厚的地层间隔(相当于赵 资奎等(1991) CGY-CGD剖面的CGD57 78m; 张显球
等(2006)大塘剖面的30 32层), 依据是狍粉组合的变化 (赵资奎等, 2017; Erben等, 1995; Stets等, 1996; Buck等, 2004).

可见尽管大塘剖面被作为中国非海相白严系-古 近系地层划分对比的标准剖面, 但是其K-Pg界线位置 仍有待进一步的确定. 本文在前人工作的基础上, 通 过总录 $(\mathrm{Hg})$ 含量、古气候演化及其与全球记录的对比 等新的视角出发, 探讨沉积环境的变化, 对南雄盆地 $\mathrm{K}$ $\mathrm{Pg}$ 界线位置进行约束, 从而判断已有的4条界线中, 哪 一条最接近真实的 $\mathrm{K}-\mathrm{Pg}$ 界线.

\section{2 地质概况及实验方法}

\section{1 地质概况}

南雄盆地 $\left(25^{\circ} 03^{\prime} \sim 25^{\circ} 16^{\prime} \mathrm{N}, 114^{\circ} 08^{\prime} \sim 114^{\circ} 40^{\prime} \mathrm{E}\right)$ 现今 年均降雨量 $1555 \mathrm{~mm}$, 年均气温 $19.6^{\circ} \mathrm{C}$, 位于中国东 南部, 大部分属于广东省北部(图1a), 向北东延伸到江 西省信丰县境内(Li等, 2010). 位于诸广山和青嶂山两 大花岗岩体之间, 是一个受北缘南雄大断裂控制的断 陷盆地, 白严纪晚期到古近纪, 南雄盆地主要受到拉 伸作用(舒良树等, 2004). 盆地内沉积了完整的上白严 统-早古新统的红色、紫红色泥岩-砂岩. 盆地周围除 了花岗岩外, 还有部分寒武系-侏罗系地层. 盆地内地 层出露良好, 总体北东走向(图1b), 向西北倾斜, 倾角 $10^{\circ} \sim 20^{\circ}$ (张显球等, 2013), 地层厚度最厚处可超过 $7 \mathrm{~km}$. 按照地层沉积物的性质, 可以划分为 2 群 9 组, 即 南雄群(长坝组, 江头组, 园圃组, 大凤组, 主田组, 浈水 组)及罗佛寨群(上湖组, 浓山组, 古城村组), 其中南雄 群为上白严统地层, 富含恐龙、恐龙蛋化石, 罗佛寨群 为古新统地层, 富含哺乳动物化石(张显球等, 2013). 根据盆地内红层沉积物的地球化学成分与周围地层岩 体成分的一致性以及盆地内砾石粗大、分选差、具棱 角状等特征, 推测盆地内的沉积物为近源堆积(舒良树 等, 2004), 且从白严纪晚期到古近纪早期, 沉积物的物 源一直比较稳定(Yan等, 2007).

从20世纪20 30年代开始, 大量的研究工作在盆地 内开展, 包括层序地层、古生物、古地磁及地球化学 等(赵资奎等, 1991, 2017; 赵资奎和严正, 2000; 张显 球等, 2000, 2006, 2013; 张显球和李罡, 2015; Zhao等, 2002; Yan等, 2007; Clyde等, 2010; Li等, 2010). 研究者 们在盆地内实测了多条标准剖面, 其中大塘剖面(图1c, 


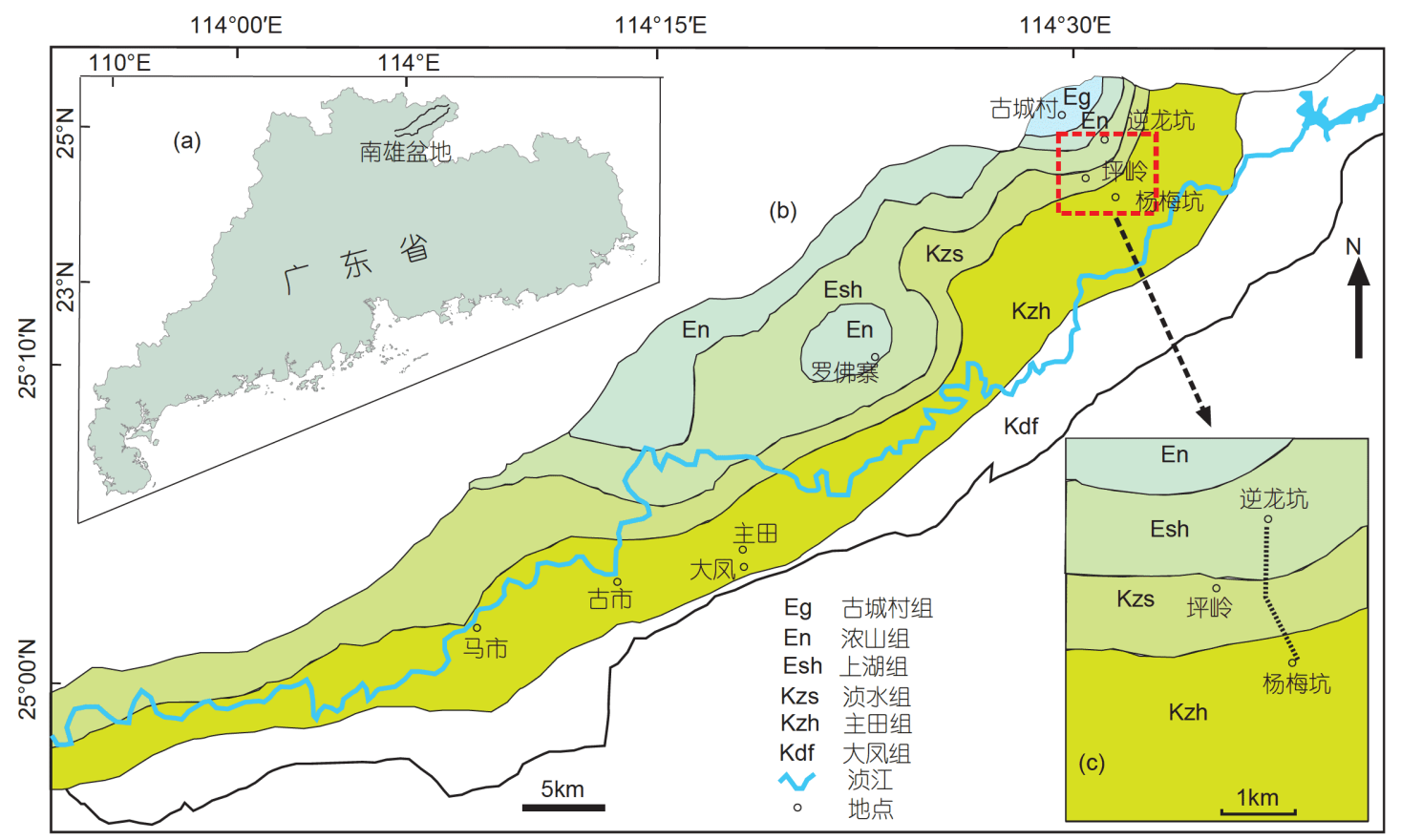

(d)

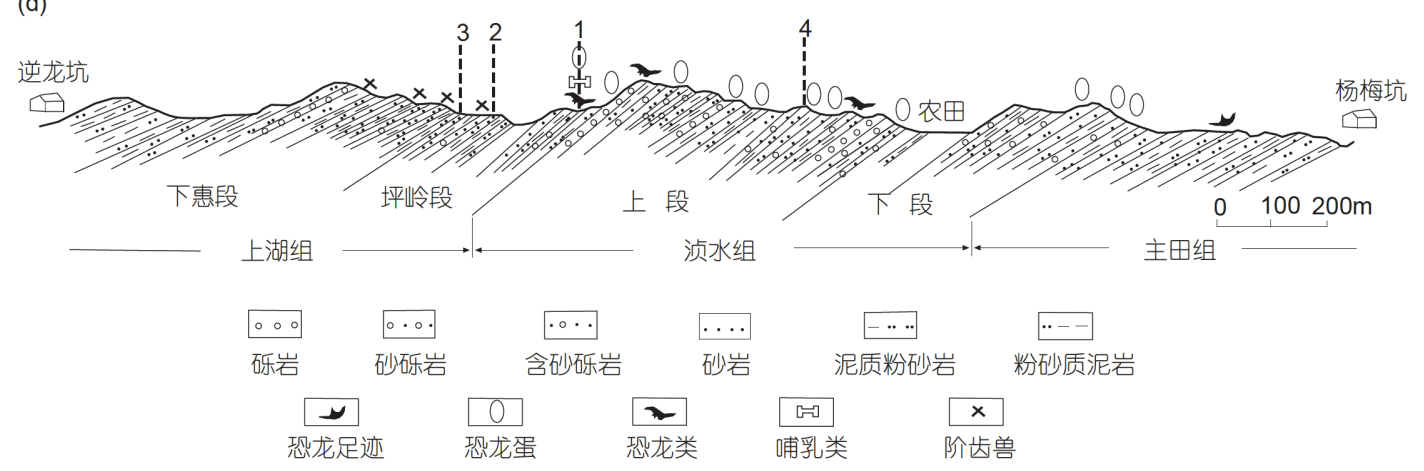

图 1 南雄盆地示意图

(a) 南雄盆地位置; (b) 南雄盆地地层(从大风组到古城村组, 修改自 Li等, 2010); (c) 大塘剖面采样路线; (d) 大塘剖面白严系-古近系地层实测剖 面(修改自张显球等, 2006), 1 4代表已有的4条界线

d)层序清楚, 化石丰富, 研究程度高. 许多科研单位和 研究者对大塘剖面进行了实测和地层划分, 多数研究 者认为大塘剖面是连续沉积、整合接触(赵资奎和严 正, 2000; 张显球等, 2006, 2013; 童永生等, 2013), 这从 我们重建的古气候记录及其与全球对比也得到证实 (详见下文). 本文采用张显球的划分方案, 该剖面起于 杨梅坑村, 止于逆龙坑村(图1c), 剖面总长度 $2300 \mathrm{~m}$, 地 层厚度近 $700 \mathrm{~m}$ (张显球等, 2006), 以粉砂质泥岩及泥质 粉砂岩为主, 夹有砂岩、砾岩. 从上至下地层依次为上 湖组 $(287.3 \mathrm{~m})$ 、浈水组 $(294.9 \mathrm{~m})$ 及主田组上部 (104.6m). 各组的岩性特征及沉积物粗细情况如下(表 1, 张显球等, 2006, 2013):

\section{2 实验方法}

将大塘剖面野外采集的样品带回实验室，自然风 干后测量其总 $\mathrm{Hg}$ 含量. 利用Hydra-C录蒸气计(美国利 曼实验室公司)和冷原子吸收光谱仪测定总 $\mathrm{Hg}$ 含量, 最低检测限为 $2 \mathrm{ppb}(1 \mathrm{ppb}=1 \mathrm{ng} / \mathrm{g})$. 测量原理是基于固体 样品中 $\mathrm{Hg}$ 的直接热蒸发 $\left(800^{\circ} \mathrm{C}\right.$ 持续 180 秒 $)$, 因此不需 要对样品进行化学预处理, 从而避免了样品制备过程 中的潜在污染. 每 5 个样品重复测量一个样品, 以确保 数据的稳定性和准确性. 总 $\mathrm{Hg}$ 含量的测试在中国科学 院南京地理与湖泊研究所完成.

选取代表性样品进行总有机碳(Total Organic Car- 
表 1 大塘剖面各组的岩性特征及沉积物粗细情况 ${ }^{\text {a) }}$

\begin{tabular}{|c|c|c|c|c|}
\hline \multicolumn{2}{|c|}{ 组段 } & 厚度(m) & 岩性特征 & 沉积物 \\
\hline \multirow{2}{*}{ 上湖组 } & 下惠段 & 199 & \multirow{2}{*}{$\begin{array}{l}\text { 暗紫、深褐色富含钻质结核的泥质粉砂岩和粉砂质泥岩, 夹薄层透 } \\
\text { 镜状含砂砾岩、粗-中粒砂岩, 局部夹绿色薄层状泥岩或页岩 }\end{array}$} & \multirow{2}{*}{ 细碎屑岩 } \\
\hline & 坪岭段 & 88.3 & & \\
\hline \multirow{2}{*}{ 浈水组 } & 上段 & 164.8 & \multirow{2}{*}{$\begin{array}{c}\text { 以粗碎屑岩发育, 灰紫色砂砾、含砾粗砂岩、中粗粒砂砾岩与红 } \\
\text { 褐、棕红色砂岩、粉砂质泥岩互层 }\end{array}$} & \multirow{2}{*}{ 粗碎屑岩 } \\
\hline & 下段 & 130.1 & & \\
\hline 主田组 & 上部 & 104.6 & $\begin{array}{c}\text { 厚层紫红、褐红色泥质粉砂岩, 间夹薄层状、条带状细砂岩, 砂岩局 } \\
\text { 部含少量砾石 }\end{array}$ & 细碎屑岩 \\
\hline
\end{tabular}

a) 改自张显球等 $(2006,2013)$

bon, TOC)测试, 将800 1000mg研磨后的干土装入 $50 \mathrm{~mL}$ 的烧杯中，每个烧杯内加入 $3 \sim 4$ 滴 $1 \mathrm{~mol} / \mathrm{L}$ 的 $\mathrm{HCL}$, 使盐酸与样品充分反应, 然后用去离子水冲洗样品至 $\mathrm{PH}$ 为中性, 将样品放入烘箱 $40^{\circ} \mathrm{C}$ 烘干后再利用土壤碳 氮元素分析仪(Elementar Vario EL III, 德国)测试样品 的TOC.

在 $\mathrm{Hg}$ 测试的基础上选择大塘剖面4条界线前后的 样品, 详细的测量磁化率(Magnetic Susceptibility, $\chi$ )、 饱和等温剩磁(Saturation Isothermal Remanent Magnetisation, SIRM)、地球化学元素含量. 把自然风干后的 样品放在研钵中轻微研磨, 称取粉末状样品装入磁测 专用无磁性塑料盒中, 用Bartington MS2磁化率仪测 试样品的 $\chi$ (测试频率为 $0.47 \mathrm{~Hz}$ ), 经质量归一后, 得到 样品的 $\chi$. 使用IM-10-30强磁仪对样品施加1T的磁场, 利用Molspin Minispin 旋转磁力仪测量得到SIRM.

使用带有金属陶瓷管的全解析Epsilon 3-XLE光 谱仪通过X射线荧光(XRF)分析测定了主要元素的组 成，并将主要元素表示为氧化物. X射线管阳极在 4 50kV加速电压下工作，最大功率 $15 \mathrm{~W}$ ，用600s 积分 时间测量 $\mathrm{Na} \sim \mathrm{K}$ 元素，每个样品测量3次，得到平均值. 每10个样品使用标准物质, 重复性大于 $95 \%$. XRF结果 用于计算化学蚀变指数 (CIA-mol), 计算公式为 CIA $=$ $\left[\mathrm{Al}_{2} \mathrm{O}_{3} /\left(\mathrm{Al}_{2} \mathrm{O}_{3}+\mathrm{CaO} *+\mathrm{Na}_{2} \mathrm{O}+\mathrm{K}_{2} \mathrm{O}\right)\right] \times 100 \%$ (Nesbitt和 Young, 1982), 其中 $\mathrm{CaO}$ *是样品硅酸盐部分中 $\mathrm{CaO}$ 的 量，本研究没有利用酸浸法去除非硅酸盐矿物，所以 采用了McLennan等(1993)提出的校正方法: 依据自然 界硅酸盐矿物中 $\mathrm{Ca}$ 和 $\mathrm{Na}$ 的平均组成, 再根据沉积样品 中 $\mathrm{CaO} / \mathrm{Na}_{2} \mathrm{O}$ 的摩尔比值来计算CIA. 若比值小于 1 , 直 接用 $\mathrm{CaO}$ 的摩尔含量来计算CIA, 若比值大于 1 , 则用 $\mathrm{Na}_{2} \mathrm{O}$ 的摩尔含量替代 $\mathrm{CaO}$ 的含量来计算CIA. $\chi$ 、 SIRM、地球化学元素含量和TOC的测试在福建师范
大学湿润亚热带生态-地理过程教育部重点实验室 完成.

\section{3 结果}

整个大塘剖面总 $\mathrm{Hg}$ 含量在1.69 15.58ppb之间, 低 于已发表的海相K-Pg剖面Hg含量(Sial等, 2013, 2016; Font等, 2016, 2018; Keller等, 2018), 这可能是由于海 相地层和陆相地层的保存条件不一样: 海相地层有机 质含量多, $\mathrm{Hg}$ 吸附在有机质上更易保存; 而当时大塘 剖面为干旱氧化环境, 不利于 $\mathrm{Hg}$ 的保存. 虽然大塘剖 面 $\mathrm{Hg}$ 含量总体较低, 但结果显示剖面存在 $\mathrm{Hg}$ 异常区域 (图2c, 2d). 其中主田组和浈水组下段 $\mathrm{Hg}$ 含量处于较低 的水平, 到了浈水组上段和上湖组坪岭段, $\mathrm{Hg}$ 含量明 显开始升高, 且表现出剧烈的峰谷波动, 之后在上湖组 下惠段又下降到与主田组及浈水组下段相当的低值. $\mathrm{Hg}$ 含量波动曲线中有四次峰值比较显著: 第一次出现 在浈水组上段, 与界线4重合, 处于 $\mathrm{Hg}$ 异常区初始位置; 第二次大致位于浈水组与上湖组交界处，靠近界线 1 , 位于 $\mathrm{Hg}$ 异常区中部; 第三次位于上湖组坪岭段, 在界 线1和界线 2 之间, 位于 $\mathrm{Hg}$ 异常区中后部; 第四次位于 上湖组下惠段底部，在界线 2 和界线 3 之间，位于 $\mathrm{Hg}$ 异 常区后部(图2a, 2c). 图2b显示大塘剖面的TOC值非常 低, 绝大部分样品含量低于 $0.1 \%$, 与前人研究结果一 致(Yan等, 2007), 整体的变化趋势与 $\mathrm{Hg}$ 呈现出一定的 反相关, 即在主田组、浈水组下段和上湖组下惠段含 量较高, 在浈水组上段和上湖组坪岭段含量较低.

从 $\chi$ 结果来看, $\chi$ 值整体较低, 但不同组段之间又有 不同的变化趋势. 浈水组 $\chi$ 变化可以分为两个相似的阶 段: 66.6 66.3Ma $\chi$ 先升后降, 66.3 66Ma $\chi$ 也表现出同 样的变化, 上升幅度比第一次的更大; 上湖组的 $\chi$ 整体 
(a)

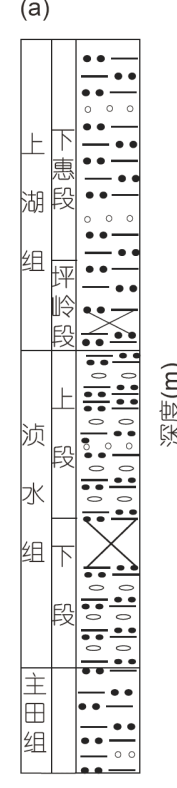

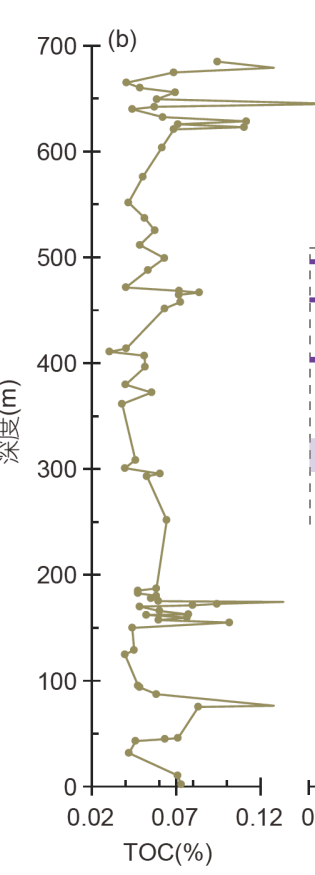

(c)

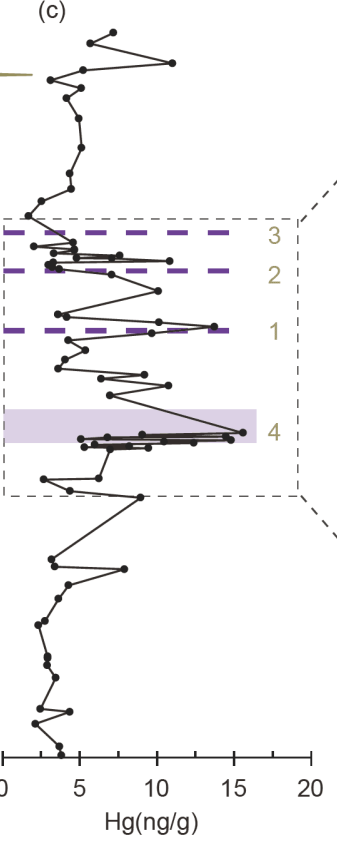

$65.4 T^{(d)}$

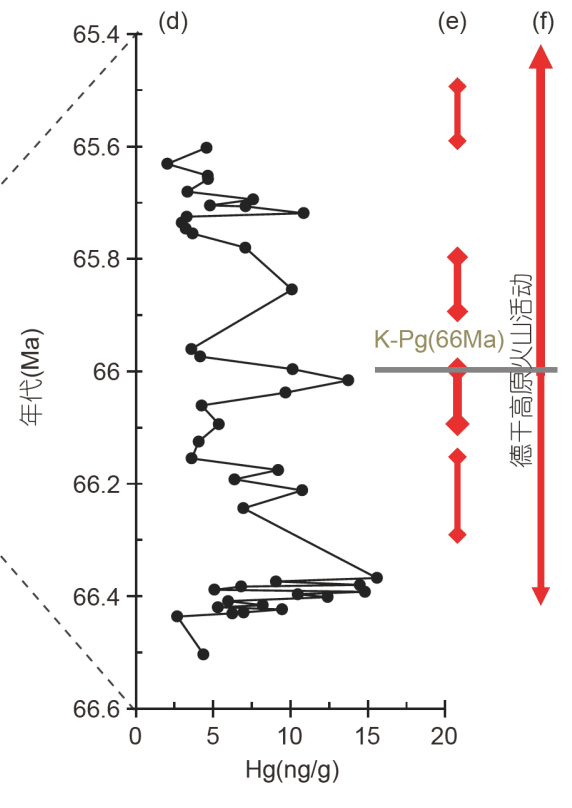

图 2 大塘剖面TOC及总 $\mathrm{Hg}$ 含量

(a) 大塘剖面岩性地层; (b) TOC曲线; (c) 总 Hg含量曲线, 紫色的虚线分别表示界线1、2、3, 紫色条表示界线4; (d) 经Clyde等 (2010)的古地磁 年代校正的Hg含量曲线; (e) Schoene等 (2019)发表的德干高原火山喷发U-Pb年代, 灰色实线表示K-Pg界线; (f) Sprain等 (2019)发表的德干高 原火山喷发 ${ }^{40} \mathrm{Ar}-{ }^{39} \mathrm{Ar}$ 年代(红色线条越粗代表喷发量越多)

上要高于浈水组, 65.96 65.85Ma由于农田的覆盖, 没 有记录, 在 $\sim 65.7 \mathrm{Ma}$ 时 $\chi$ 显著降低, 前后 $\chi$ 的两个峰值可 以与 $\delta^{13} \mathrm{C}_{\mathrm{carb}}$ 的两次负偏较好的对应. 土壤钙结核的碳 氧同位素数据来自Clyde等(2010)和王尹(2012)(图3b, $3 \mathrm{c}$ ), 其中几个显著的变化是 $66.2 \mathrm{Ma}$ 附近的 $\delta^{18} \mathrm{O}_{\text {carb }}$ 正偏 和 $\delta^{13} \mathrm{C}_{\mathrm{carb}}$ 的负偏; $65.7 \mathrm{Ma}$ 附近 $\delta^{13} \mathrm{C}_{\mathrm{carb}}$ 的两次显著负偏.

\section{4 讨论}

一般来说，国际上对于K-Pg的界定依据有 5 个: 浮游有孔虫大灭绝; (2) Danian物种的首次出现; (3) $\delta^{13} \mathrm{C}$ 负漂移; (4) 铱(Ir) 异常; (5) 红色粘土层(Keller等, 2018). 在没有生物依据的前提下，后三个依据不能作 为判定K-Pg的唯一标准(Keller, 2011). 从这些标准来 看, 更多的是对海相地层的界定. 而对于北美地区陆相 地层来说, K-Pg大多是根据孢粉资料确定的(刘耕武等, 2009). 借鉴北美地区的研究经验, Erben等(1995)根据孢 粉组合的变化, 将K-Pg置于浈水组上段的下部20余米 厚地层中, 并通过沉积速率推测其持续了 $50 \mathrm{kyr}$ (界线 4, 图3). 此后中国研究者多次在该界线附近采集样品
进行孢粉鉴定，但是并没有发现可以用来说明界线问 题的孢粉组合变化(刘耕武等, 2009). 此外, 南雄盆地 当时的古气候为热带干旱半干旱，而北美地区同期为 湿润气候带, 古气候的差异导致植被类型及保存条件 存在差异, 因此很有可能造成南雄盆地的孢粉资料无 法与北美地区直接进行对比，会造成运用狍粉资料确 定K-Pg的困难(刘耕武等, 2009; 李伟同等, 2014).

最近, Clyde等(2010)通过古地磁、同位素及生物 化石等, 发现在他们所划分的上湖组底界(灰黄色底砾 岩)附近, $\delta^{13} \mathrm{C}$ 发生显著负漂移, 且界线位于古地磁 $\mathrm{C} 29 \mathrm{r}$ 的上面 $2 / 3$ 处, 这与国际大多数的 K-Pg位置吻合, 并结合非鸟类恐龙的灭绝及对比全球Danian期哺乳动 物的兴起时间, 从而再次确认了其作为K-Pg的可行性 (界线1, 图3). 然而, 赵资奎等(2017)认为南雄盆地的 古地磁结果存在争议, 需要进一步的研究, 仍更倾向于 他们划分的孢粉界线(界线4). 由此可见，最近发表的 成果集中于对界线 1 和界线 4 的讨论，虽然补充了一些 证据, 但还是无法确定哪条界线更接近于真实的 K-Pg, 所以，对于K-Pg的界定仍然需做很多的工作. 鉴于此, 对南雄盆地K-Pg的界定标准也应该有所侧重和创新, 


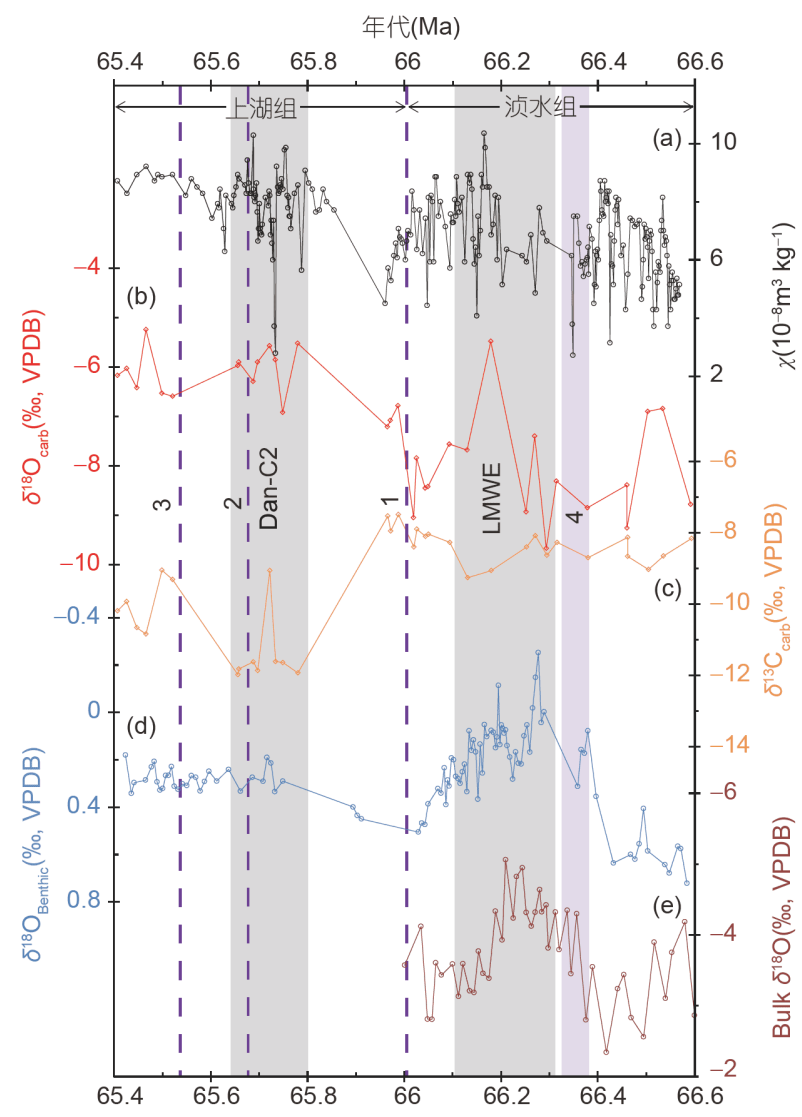

图 3 大塘剖面浈水组上段到上湖组坪岭段古气候演化

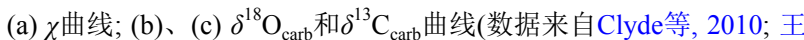
尹, 2012); (d) 太平洋Ocean Drilling Program 1209 站点 $\delta^{18} \mathrm{O}_{\text {benthic }}$ 曲线 (Westerhold等, 2011); (e) 突尼斯Elles剖面 $\delta^{18} \mathrm{O}$ 曲线(Thibault等, 2015); 其中, 紫色的虚线分别表示界线1、2、3，紫色条表示界线4, 灰色条表示LMWE、Dan-C2事件. 年代采用Clyde等 (2010)的古地 磁结果

除了参考常规的鉴定标准外，新标准新手段的应用也 许可以为问题的解决提供新的思路.

$\mathrm{Hg}$ 作为自然界唯一一种挥发性金属元素，在地质 时期常常伴随火山活动被大量排放到大气中(Pyle和 Mather, 2003; Zambardi等, 2009), Hg在大气中的停留 时间为 $0.5 \sim 2$ 年, 保证其全球分布的同时也能快速沉降 到陆地表面及水体中保存下来(Pyle和Mather，2003; Witt等, 2008; Bagnato等, 2011), 被成功地用作重建古 火山活动的标志物(Grasby等，2015，2019; Shen等, 2019a). 近年来，越来越多的研究利用Hg将地质时期 的生物大灭绝事件与火山活动联系起来(例如Shen等, 2019a，2019b，2019c)，也有研究发现K-Pg界线附近也 出现显著Hg浓度异常, 并将其归因于德干高原火山的
喷发(Silva等, 2013; Sial等, 2013, 2014; Font等, 2016, 2018; Keller等, 2018, 2020). 尽管德干高原火山喷发的 精确年代有待更多的工作来确定，但是最新的年代结 果显示Deccan Traps是在小于1个百万年的时间内完 成喷发(66.3 65.5Ma，U-Pb年代，Schoene等，2019; 66.413 65.422Ma, ${ }^{40} \mathrm{Ar}-{ }^{39} \mathrm{Ar}$ 年代, Sprain等, 2019, 图2) 且整个喷发过程确实跨越了 $\mathrm{K}-\mathrm{Pg}$ 界线, 为利用 $\mathrm{Hg}$ 研究 $\mathrm{K}-\mathrm{Pg}$ 界线提供了可能.

在前人的研究中, $\mathrm{Hg}$ 经常依附于某种物质保存下 来, 比如有机质、硫化物等(Sial等, 2013; Font等, 2016; Grasby等, 2019; Shen等, 2019d, 2020). 而本研究剖面 的TOC浓度基本都低于 $0.1 \%$, 低于可以校正的TOC最 低值 $(0.1 \%$, Shen等，2019a)，且TOC整体表现出与Hg 含量反相关的趋势(图2), 表明南雄盆地的 $\mathrm{Hg}$ 并不是保 存在TOC中. 此外, 从沉积物粗细来看, $\mathrm{Hg}$ 含量高的部 位既包含粗碎屑沉积(浈水组上段)也有细碎屑沉积 (上湖组坪岭段)，表明沉积物的“粒控效应”对 $\mathrm{Hg}$ 含 量也没有影响. 将样品中 $\mathrm{Al}_{2} \mathrm{O}_{3} 、 \mathrm{SiO}_{2} 、 \mathrm{CaO} 、 \mathrm{MgO}$ 、

$\mathrm{Fe}_{2} \mathrm{O}_{3} 、 \mathrm{~K}_{2} \mathrm{O}$ 这几种主要氧化物及 $\mathrm{Ti}$ 的值与总 $\mathrm{Hg}$ 含量 作相关性分析, 从散点图结果来看, $\mathrm{Hg}$ 与这些氧化物 都不存在显著的相关关系(图4), 这说明本研究中 $\mathrm{Hg}$ 的 来源最有可能是经大气直接沉降而不依附于某种特定 物质, 可以反映火山活动排放到大气中 $\mathrm{Hg}$ 含量的变化. 大塘剖面 $\mathrm{Hg}$ 异常区大约持续了 $0.8 \mathrm{Ma}(66.4 \sim 65.6 \mathrm{Ma}$, 图 2d), 元素异常时间长, 应该是由大型火山喷发造成的. 目前南雄盆地内部测得代表火山活动的年代有两期, 一是以舒良树(2004)为代表测得的位于主田组中部的 吊当排的玄武岩年龄 $(96 \pm 1) \mathrm{Ma}$ ，二是在浓山组中部发 现的火山灰层，其年代为 $60 \mathrm{Ma}$ (童永生等，2013), 这 两个年代与 $\mathrm{Hg}$ 异常区间的年代不符; 而中国华南地区 的大规模的火山活动集中在早白严世，并没有在白严 纪晚期发现大型的火成岩省( $\mathrm{Li}$ 等，2019). 这样看来, 大塘剖面的 $\mathrm{Hg}$ 异常是受到世界其他地区火山活动的 影响, 而白严纪晚期-古近纪早期规模和影响最大的火 山活动是德干高原火山活动, 并且大塘剖面 $\mathrm{Hg}$ 异常区 间与德干高原火山活动时间大致符合(图 $2 \mathrm{~d}, 2 \mathrm{e}, 2 \mathrm{f}$ ). 因 此, 可以将南雄盆地 $\mathrm{Hg}$ 异常和德干高原火山活动联系 起来.

Schoene等(2019)及Sprain等(2019)的年代学结果 显示 K-Pg大约位于德干高原火山喷发的中期(图2e, $2 \mathrm{f}$ ), 南雄盆地 4 条K-Pg界线中只有界线1位于 $H g$ 异常区 

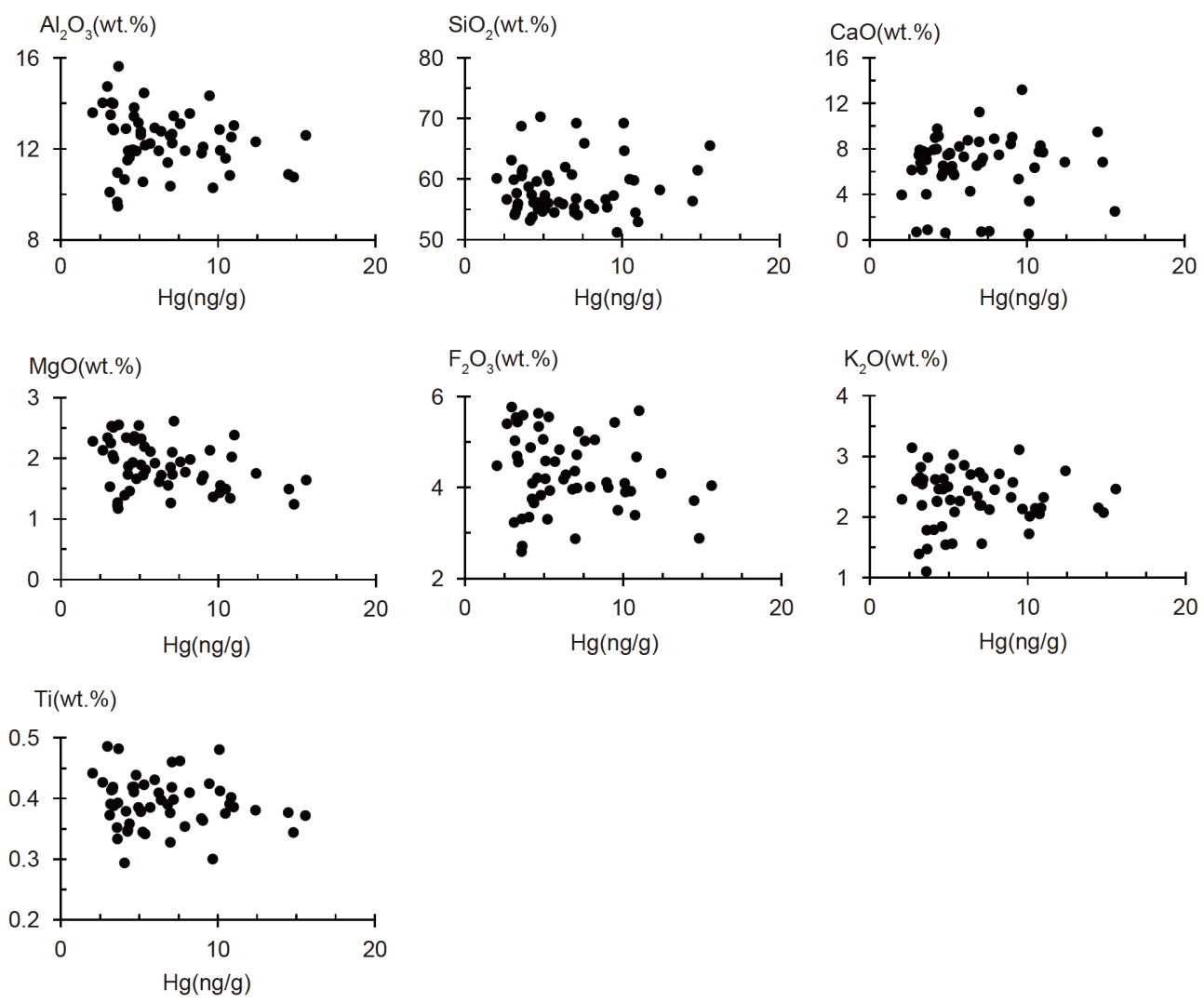

图 4 总 $\mathrm{Hg}$ 含量与主要氧化物之间的关系

域的偏中间位置，也就是德干高原火山喷发的中期(图 2c). 而其余3条界线的位置都有或多或少的偏差: 界线 2 靠近 $\mathrm{Hg}$ 异常区域的结束处, 界线3并不在 $\mathrm{Hg}$ 异常区间 内, 界线4位于 $\mathrm{Hg}$ 异常区间开始处(图2c). 所以, 从总 $\mathrm{Hg}$ 含量来看, 界线 1 附近既出现了 $\mathrm{Hg}$ 含量异常高值, 在时 间上又能与德干高原火山喷发的时间进行很好的对 比, 符合全球 $\mathrm{K}-\mathrm{Pg}$ 界线与德干高原火山喷发的关系.

成壤碳酸盐 $\delta^{18} \mathrm{O}$ 主要取决于大气降水的氧同位素 值、大气温度及蒸发作用等因素(Cerling等，1989; Cerling和Quade, 1993; Quade等, 2007), 大量的地质记 录揭示出白严纪古土壤中成壤碳酸盐 $\delta^{18} \mathrm{O}$ 可以用来反 映其形成时期的大气古温度(比如 Gao等, 2015), 南雄 盆地 $\delta^{18} \mathrm{O}_{\mathrm{carb}}$ (图3b)与全球同期深海记录(图3d)趋势一 致, 证明了 $\delta^{18} \mathrm{O}_{\text {carb }}$ 代表了古温度的变化. 此外, 南雄盆 地大塘剖面 $\chi$ (图3a)与 $\delta^{18} \mathrm{O}_{\text {carb }}$ (图3b)以及同时期的深海 记录(图3d)也可以形成很好的对比，证实其记录了与 全球一致的古气候演化, $\mathrm{Ma}$ 等(2018)据此提出 $\chi$ 记录古 气候的机制——加强版的“成壤说”，即成壤过程中生

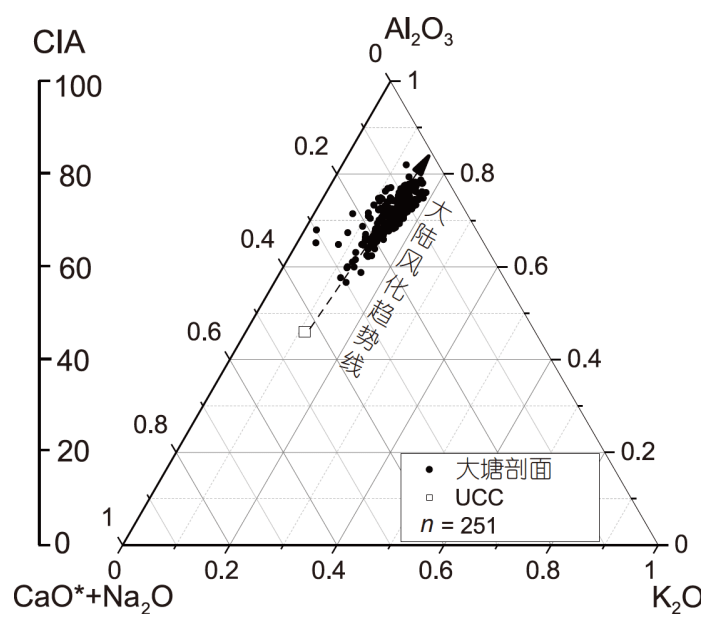

图 5 大塘剖面沉积物化学组成A-CN-K图

成的磁性矿物在热带干旱-半干旱气候背景下, 经历低 温氧化作用, 彻底转变为最终产物一一赤铁矿, 且赤铁 矿的含量与成壤强度有关, 因此, $\chi$ 在研究区可以作为 可靠的古气候代用指标. 一般来说, SIRM与磁性矿物 
的浓度相关(Thompson和Oldfield, 1986), CIA值可以指 示风化成壤作用的强弱(Nesbitt和Young，1982，1984). 为了探究CIA指标指示本研究区风化作用的可靠性, 绘制了 $\mathrm{A}-\mathrm{CN}-\mathrm{K}\left(\mathrm{Al}_{2} \mathrm{O}_{3}-\mathrm{CaO} *+\mathrm{Na}_{2} \mathrm{O}-\mathrm{K}_{2} \mathrm{O}\right.$ ) 图 (图5), 该图 常常被用来估测大陆风化趋势(Nesbitt等，1980). 根据 元素活动性顺序可以把化学风化过程分为: 早期脱 $\mathrm{Ca}$ 和 $\mathrm{Na}$ ，中期脱 $\mathrm{K}$, 晚期脱 $\mathrm{Si}$ 阶段(Nesbitt等，1980). 从A$\mathrm{CN}-\mathrm{K}$ 图中可以看出, 所有样品点在图中平行于 $\mathrm{A}-\mathrm{CN}$ 边，这表明沉积物处于化学风化早期脱 $\mathrm{Ca}$ 和 $\mathrm{Na}$ 的阶 段, 且沉积物未发生钾的交代作用，即在成岩过程中 没有代入新的钾元素. 大部分样品的CIA值介于 $65 \sim 80$ 之间, 表明沉积物为中等化学风化程度. 样品主要沿上 地壳平均组成的风化趋势线分布，表明大塘剖面沉积 物的来源区比较广泛，且经过了充分物质循环混合 (Taylor和McLennan, 1985)，这与我们前期(Ma等, 2018)利用稀土元素的配比模式得到物源稳定且充分 混合的结论一致，因此CIA值在本研究区可以指示风 化成壤作用的强弱. 图6中 $\chi$ 与SIRM、CIA呈现出显著 的正相关关系, 进一步说明 $\chi$ 的变化受控于风化成壤过 程中生成的赤铁矿含量, 为 $\chi$ 在研究区古气候指示的可 靠性提供了更多的证据支持.

目前全球已有的大量古气候重建工作指示了在马 斯特里赫特期末期发生了一次变暖事件( 66.2 Ma, Late Maastrichtian warming event, LMWE)(Li和Keller, 1998; Abramovich等，2003; Nordt等，2003; Wilf等, 2003; Thibault和Gardin, 2010; Woelders等, 2017, 2018; Hull等, 2020), 并将其归因于德干高原火山喷发的 $\mathrm{CO}_{2}$ 导致的温室效应(Li和Keller, 1998; Nordt等, 2003; Wilf 等, 2003; Thibault等, 2015; Woelders等, 2018), 该暖事
件之后到 $\mathrm{K}-\mathrm{Pg}$ 界线全球古气候又发生显著的变冷. 图 3 显示了研究区 4 条界线前后的细致的古气候演化过 程：在66.2 Ma期间， $\chi$ 为高值区、 $\delta^{18} \mathrm{O}_{\text {carb }}$ 显著正偏、 $\delta^{13} \mathrm{C}_{\mathrm{carb}}$ 显著负偏，指示了一次明显的气候变暖，对应 于全球LMWE事件; 之后到 $66 \mathrm{Ma}, \chi$ 降低、 $\delta^{18} \mathrm{O}_{\text {carb }}$ 负 偏, 指示了快速的降温, 这一变化的细节可以很好的 与多地的记录进行对比(如全球层型剖面一一突尼斯 Elles剖面 $\delta^{18} \mathrm{O}$ 曲线, Thibault等, 2015, 图3e). 对比南雄 盆地4条K-Pg界线及LMWE事件的大致位置，可以发 现只有界线 1 的位置最符合全球记录; 界线 4 位于 LMWE事件之前; 界线 2 附近 $\chi$ 表现为双峰，对应着 $\delta^{13} \mathrm{C}_{\text {carb }}$ 的两次负漂移，可能对应于 $\mathrm{K}-\mathrm{Pg}$ 之后的热事件 Dan-C2 event (Dan-C2, Quillévéré等, 2008; Coccioni 等, 2010, 图3); 界线3位于Dan-C2事件之后. 因此, 从古 气候记录的角度看, 也只有界线 1 最接近真实的 $\mathrm{K}-\mathrm{Pg}$ 界线.

\section{5 结论}

本文在前人研究的基础上，对大塘剖面总 $\mathrm{Hg}$ 含 量、古气候演化进行了研究, 为确定南雄盆地 $\mathrm{K}-\mathrm{Pg}$ 界 线提供新的证据:

(1) 大塘剖面总 $\mathrm{Hg}$ 含量在浈水组上段和上湖组坪 岭段明显升高，且表现出剧烈的峰谷波动,记录了德干 高原火山活动. 结合最新的德干高原火山喷发年代结 果，4条界线中，只有界线1所处的位置符合 K-Pg界线 与火山活动的关系.

(2) 南雄盆地记录了LMWE事件, 界线1位于 LMWE事件之后，且界线 1 与LMWE之间记录了短

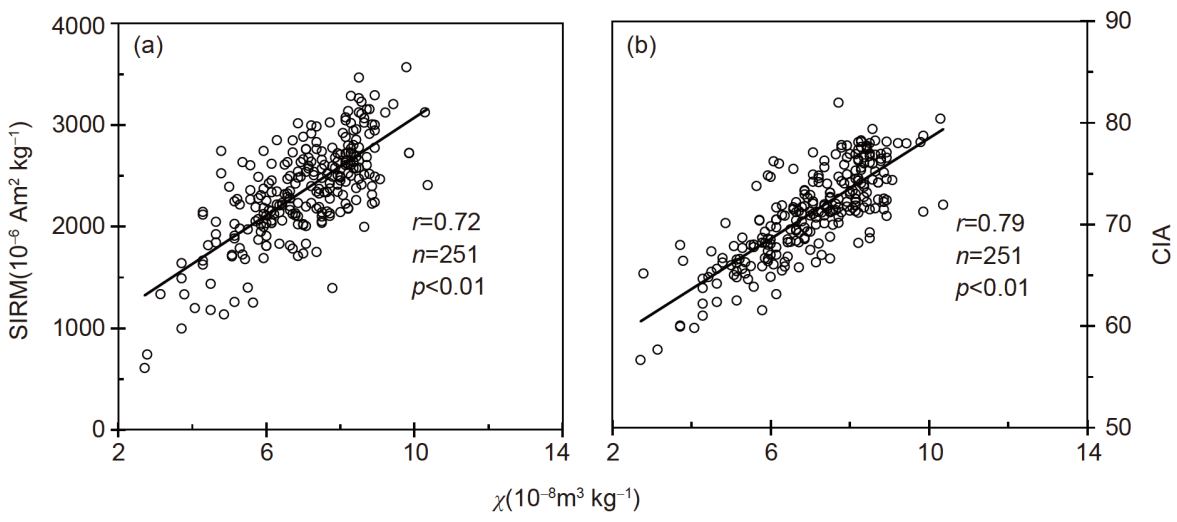

图 $6 \chi$ 与 SIRM(a)、 $\chi$ 与 CIA(b) 的散点图 
暂、快速的变冷事件, 与全球其他地区记录相符合. 总的来说，界线 1 无论是在总 $\mathrm{Hg}$ 含量指示的古火 山活动记录上，还是在古气候演化记录上，都最接近 南雄盆地真实的 $\mathrm{K}-\mathrm{Pg}$ 界线.

致谢感谢两位审稿专家提出的建设性修改意见, 感谢 中国科学院南京地理与湖泊研究所张恩楼研究员与朱育 新副研究员对总永含量测试的支持和帮助.

\section{参考文献}

江小均, 柳永清, 姬书安, 张兴辽, 徐莉, 贾松海, 吕君昌, 袁崇喜, 李 明. 2011. 豫西橸川-潭头盆地含脊椎动物化石地层序列和 $\mathrm{K} / \mathrm{T}$ 界 线初步研究. 中国科学: 地球科学, 41: 738-744

李莎, 高琴琴, 张翼翼, 屈海英, 王曼艳, 万晓樵. 2013. 松辽盆地“松 科1井(北孔)”晚白严世晚期至古新世早期轮藻化石组合. 微体古 生物学报, 30: 1-16

李伟同, 刘耕武, Braman D R, 曹卫生, 陈钦保, Brinkman D. 2010. 湖 北当阳陆相白严系-古近系界线层型问题值得深入研究. 地层学 杂志, 34: 187-206

李伟同, 刘耕武, Braman D R, 李艺斌, 曹卫生, Brinkman D, 沈娇, 陈 钦保. 2014. 中国新生界第一阶潜在的层型剖面. 中国科学: 地球 科学, 44: 634-641

刘耕武, Braman, D R, 李伟同, Brinkman D. 2009. 北美西部陆相白严 系-古近系界线孢粉学特征简介——兼评中国东部白严系-古近 系界线研究. 地层学杂志, 33: 18-34

凌秋贤, 张显球, 林建南. 2005. 南雄盆地白严纪-古近纪地层研究进 展. 地层学杂志, 29(增刊): 596-601

舒良树, 邓平, 王涁, 谭正中, 余心起, 孙岩. 2004. 南雄-诸广地区晚中 生代盆山演化的岩石化学、运动学与年代学制约. 中国科学 $\mathrm{D}$ 辑: 地球科学, 34: 1-13

孙革. 2014. 黑龙江嘉荫晚白严世-古新世生物群、K-Pg界线及恐龙 灭绝. 上海: 上海科技教育出版社. 188

童永生, 李曼英, 李茜. 2002. 广东南雄盆地白严系-古近系界线. 地质 通报, 21: 668-674

童永生, 李茜, 王元青. 2013. 中国早古近纪陆相地层划分框架研究. 地层学杂志, 37: 428-440

王尹. 2012. 南雄盆地晚白严世-早古新世古气候变化. 硕士学位论 文. 南京: 南京大学. 1-60

王尹, 李祥辉, 周勇, 刘玲. 2015. 南雄盆地晚白严世-古新世陆源沉积 组份变化的古气候指示. 沉积学报, 33: 116-123

席党鹏, 万晓樵, 李国彪, 李罡. 2019. 中国白严纪综合地层和时间框 架. 中国科学: 地球科学, 499: 257-288

余汶, 顾和林, 张显球. 1990. 广东南雄盆地晚白严世-早第三纪非海 相腹足类组合序列. 古生物学报, 29: 160-182, 263-266

赵资奎, 叶捷, 李华梅, 赵振华, 严正. 1991. 广东省南雄盆地白严系-
第三系交界恐龙绝灭问题. 古脊椎动物学报, 29: 1-12

赵资奎, 严正. 2000. 广东南雄盆地白严系-第三系界线剖面恐龙蛋壳 稳定同位素记录: 地层及古环境意义. 中国科学 $\mathrm{D}$ 辑: 地球科学, 30: 135-141

赵资奎, 毛雪摸, 柴之芳, 杨高创, 张福威, 严正. 2009. 广东省南雄盆 地白严纪-古近纪 $(\mathrm{K} / \mathrm{T})$ 过渡时期地球化学环境变化和恐龙灭绝: 恐龙蛋化石提供的证据. 科学通报, 54: 201-209

赵资奎, 叶捷, 王强. 2017. 南雄盆地白严纪-古近纪交界恐龙灭绝和 哺乳动物复苏. 科学通报, 17: 1869-1881

张显球. 1984. 南雄盆地坪岭剖面罗佛寨群的划分及其生物群. 地层 学杂志, 8: 239-254

张显球. 1992. 广东南雄盆地上湖组介形类动物群及白严-第三系界

线. 古生物学报, 31: 678-702, 773-776

张显球, 黎三松, 李永丰. 2000. 南雄盆地西部地区地层研究新进展.

广东地质, 15: 9-18

张显球, 凌秋贤. 2004. 南雄盆地白严-古近系( $\mathrm{E} / \mathrm{K})$ 界线研究现状. 第 九届中国古脊椎动物学学术年会. $45-52$

张显球, 林建南, 李罡, 凌秋贤. 2006. 南雄盆地大塘白严系-古近系界 线剖面研究. 地层学杂志, 30: 327-340

张显球, 张喜满, 侯明才, 李罡, 黎汉明. 2013. 南雄盆地红层岩石地 层划分. 地层学杂志, 37: 57-67

张显球, 李罡. 2015. 南雄盆地上湖组坪岭段的时代探讨. 地层学杂 志, 39: 74-80

Abramovich S, Keller G, Stüben D, Berner Z. 2003. Characterization of late Campanian and Maastrichtian planktonic foraminiferal depth habitats and vital activities based on stable isotopes. Palaeogeogr Palaeoclimatol Palaeoecol, 202: 1-29

Bagnato E, Aiuppa A, Parello F, Allard P, Shinohara H, Liuzzo M, Giudice G. 2011. New clues on the contribution of Earth's volcanism to the global mercury cycle. Bull Volcanol, 73: 497-510

Buck B J, Hanson A D, Hengst R A, Shu-Sheng H. 2004. “Tertiary dinosaurs" in the Nanxiong Basin, Southern China, are reworked from the Cretaceous. J Geol, 112: 111-118

Cerling T E, Quade J, Wang Y, Bowman J R. 1989. Carbon isotopes in soils and palaeosols as ecology and palaeoecology indicators. Nature, 341: 138-139

Cerling T E, Quade J. 1993. Stable carbon isotopes in soil carbonates. In: Swart P K, Lohmann K C, McKenzie J, Savin S, eds. Climate Change in Continental Isotopic Records. Washington DC: American Geophysical Union

Clyde W C, Ting S, Snell K E, Bowen G J, Tong Y, Koch P L, Li Q, Wang Y. 2010. New paleomagnetic and stable-isotope results from the Nanxiong Basin, China: Implications for the K/T boundary and the timing of Paleocene mammalian turnover. J Geol, 118: 131-143

Coccioni R, Frontalini F, Bancalà G, Fornaciari E, Jovane L, Sprovieri M. 2010. The Dan-C2 hyperthermal event at Gubbio (Italy): Global 
implications, environmental effects, and cause(s). Earth Planet Sci Lett, 297: 298-305

Erben H K, Ashraf A R, Bohm H, Hambach U, Krumsiek K, Stets J, Thein J, Wurster P. 1995. Die Kreide/Tertiar-Grenze im NanxiongBecken (Kontinentalfazies, Sudostchina). Erdwissenschaftliche Forschung, 32: 1-245

Font E, Adatte T, Sial A N, Drude de Lacerda L, Keller G, Punekar J. 2016. Mercury anomaly, Deccan volcanism, and the end-Cretaceous mass extinction. Geology, 44: 171-174

Font E, Adatte T, Andrade M, Keller G, Mbabi Bitchong A, Carvallo C, Ferreira J, Diogo Z, Mirão J. 2018. Deccan volcanism induced highstress environment during the Cretaceous-Paleogene transition at Zumaia, Spain: Evidence from magnetic, mineralogical and biostratigraphic records. Earth Planet Sci Lett, 484: 53-66

Gao Y, Ibarra D E, Wang C, Caves J K, Chamberlain C P, Graham S A, Wu H. 2015. Mid-latitude terrestrial climate of East Asia linked to global climate in the Late Cretaceous. Geology, 43: 287-290

Grasby S E, Beauchamp B, Bond D P G, Wignall P, Talavera C, Galloway J M, Piepjohn K, Reinhardt L, Blomeier D. 2015. Progressive environmental deterioration in northwestern Pangea leading to the latest Permian extinction. Geol Soc Am Bull, 127: 1331-1347

Grasby S E, Them Ii T R, Chen Z, Yin R, Ardakani O H. 2019. Mercury as a proxy for volcanic emissions in the geologic record. Earth-Sci Rev, 196: 102880

Hull P M, Bornemann A, Penman D E, Henehan M J, Norris R D, Wilson P A, Blum P, Alegret L, Batenburg S J, Bown P R, Bralower T J, Cournede C, Deutsch A, Donner B, Friedrich O, Jehle S, Kim H, Kroon D, Lippert P C, Loroch D, Moebius I, Moriya K, Peppe D J, Ravizza G E, Röhl U, Schueth J D, Sepúlveda J, Sexton P F, Sibert E C, Śliwińska K K, Summons R E, Thomas E, Westerhold T, Whiteside J H, Yamaguchi T, Zachos J C. 2020. On impact and volcanism across the Cretaceous-Paleogene boundary. Science, 367: 266-272

Keller G. 2011. Defining the Cretaceous-Tertiary boundary: A practical guide and return to first principles. In: Keller G, Adatte T, eds. The End-Cretaceous Mass Extinction and the Chicxulub Impact in Texas. Tulsa: Society for Sedimentary Geology. 23-42

Keller G, Mateo P, Punekar J, Khozyem H, Gertsch B, Spangenberg J, Bitchong A M, Adatte T. 2018. Environmental changes during the Cretaceous-Paleogene mass extinction and Paleocene-Eocene Thermal Maximum: Implications for the Anthropocene. Gondwana Res, 56: 69-89

Keller G, Mateo P, Monkenbusch J, Thibault N, Punekar J, Spangenberg J E, Abramovich S, Ashckenazi-Polivoda S, Schoene B, Eddy M P, Samperton K M, Khadri S F R, Adatte T. 2020.
Mercury linked to Deccan Traps volcanism, climate change and the end-Cretaceous mass extinction. Glob Planet Chang, 194: 103312

Li G, Hirano H, Batten D J, Wan X, Willems H, Zhang X. 2010. Biostratigraphic significance of spinicaudatans from the Upper Cretaceous Nanxiong Group in Guangdong, South China. Cretac Res, 31: 387-395

Li L, Keller G. 1998. Abrupt deep-sea warming at the end of the Cretaceous. Geology, 26: 995-998

Li X, Xu W, Liu W, Zhou Y, Wang Y, Sun Y, Liu L. 2013. Climatic and environmental indications of carbon and oxygen isotopes from the Lower Cretaceous calcrete and lacustrine carbonates in Southeast and Northwest China. Palaeogeogr Palaeoclimatol Palaeoecol, 385: $171-189$

Li X, Zhang C, Li Y, Wang Y, Liu L. 2019. Refined chronostratigraphy of the late Mesozoic terrestrial strata in South China and its tectonostratigraphic evolution. Gondwana Res, 66: 143-167

Ma M, Liu X, Wang W. 2018. Palaeoclimate evolution across the Cretaceous-Palaeogene boundary in the Nanxiong Basin (SE China) recorded by red strata and its correlation with marine records. Clim Past, 14: 287-302

McLennan S M, Hemming S, McDaniel D K, Hanson G N. 1993. Processes controlling the composition of clastic sediments. Spec Pap Geol Soc Am, 284: 21-40

Molina J M, Vera J A, Aguado R. 2006. Reworked microcodium calcarenites interbedded in pelagic sedimentary rocks (Paleocene, Subbetic, Southern Spain): Paleoenvironmental reconstruction. Spec Pap Geol Soc Am, 416: 189-202

Nesbitt H W, Markovics G, price R C. 1980. Chemical processes affecting alkalis and alkaline earths during continental weathering. Geochim Cosmochim Acta, 44: 1659-1666

Nesbitt H W, Young G M. 1982. Early Proterozoic climates and plate motions inferred from major element chemistry of lutites. Nature, 299: $715-717$

Nesbitt H W, Young G M. 1984. Prediction of some weathering trends of plutonic and volcanic rocks based on thermodynamic and kinetic considerations. Geochim Cosmochim Acta, 48: 1523-1534

Nordt L, Atchley S, Dworkin S. 2003. Terrestrial evidence for two greenhouse events in the Latest Cretaceous. GSA Today, 13: 4-9

Pyle D M, Mather T A. 2003. The importance of volcanic emissions for the global atmospheric mercury cycle. Atmos Environ, 37: 51155124

Quade J, Rech J A, Latorre C, Betancourt J L, Gleeson E, Kalin M T K. 2007. Soils at the hyperarid margin: The isotopic composition of soil carbonate from the Atacama Desert, Northern Chile. Geochim Cosmochim Acta, 71: 3772-3795

Quillévéré F, Norris R D, Kroon D, Wilson P A. 2008. Transient ocean 
warming and shifts in carbon reservoirs during the early Danian. Earth Planet Sci Lett, 265: 600-615

Renne P R, Deino A L, Hilgen F J, Kuiper K F, Mark D F, Mitchell W S, Morgan L E, Mundil R, Smit J. 2013. Time scales of critical events around the Cretaceous-Paleogene boundary. Science, 339: 684-687

Schoene B, Eddy M P, Samperton K M, Keller C B, Keller G, Adatte T, Khadri S F R. 2019. U-Pb constraints on pulsed eruption of the Deccan Traps across the end-Cretaceous mass extinction. Science, 363: $862-866$

Schulte P, Alegret L, Arenillas I, Arz J A, Barton P J, Bown P R, Bralower T J, Christeson G L, Claeys P, Cockell C S, Collins G S, Deutsch A, Goldin T J, Goto K, Grajales-Nishimura J M, Grieve R A F, Gulick S P S, Johnson K R, Kiessling W, Koeberl C, Kring D A, Macleod K G, Matsui T, Melosh J, Montanari A, Morgan J V, Neal C R, Norris R D, Pierazzo E, Ravizza G, Rebolledo-Vieyra M, Reimold W U, Robin E, Salge T, Speijer R P, Sweet A R, UrrutiaFucugauchi J, Vajda V, Whalen M T, Willumsen P S. 2010. Response-Cretaceous Extinctions. Science, 328: 975-976

Shen J, Algeo T J, Planavsky N J, Yu J, Feng Q, Song H, Song H, Rowe H, Zhou L, Chen J. 2019a. Mercury enrichments provide evidence of Early Triassic volcanism following the end-Permian mass extinction. Earth-Sci Rev, 195: 191-212

Shen J, Chen J, Algeo T J, Yuan S, Feng Q, Yu J, Zhou L, O’Connell B, Planavsky N J. 2019b. Evidence for a prolonged Permian-Triassic extinction interval from global marine mercury records. Nat Commun, 10: 1563

Shen J, Yu J, Chen J, Algeo T J, Xu G, Feng Q, Shi X, Planavsky N J, Shu W, Xie S. 2019c. Mercury evidence of intense volcanic effects on land during the Permian-Triassic transition. Geology, 47: 11171121

Shen J, Algeo T J, Chen J, Planavsky N J, Feng Q, Yu J, Liu J. 2019d Mercury in marine Ordovician/Silurian boundary sections of South China is sulfide-hosted and non-volcanic in origin. Earth Planet Sci Lett, 511: 130-140

Shen J, Feng Q, Algeo T J, Liu J, Zhou C, Wei W, Liu J, Them Ii T R, Gill B C, Chen J. 2020. Sedimentary host phases of mercury (Hg) and implications for use of $\mathrm{Hg}$ as a volcanic proxy. Earth Planet Sci Lett, 543: 116333

Sial A N, Lacerda L D, Ferreira V P, Frei R, Marquillas R A, Barbosa J A, Gaucher C, Windmöller C C, Pereira N S. 2013. Mercury as a proxy for volcanic activity during extreme environmental turnover: The Cretaceous-Paleogene transition. Palaeogeogr Palaeoclimatol Palaeoecol, 387: 153-164

Sial A N, Chen J, Lacerda L D, Peralta S, Gaucher C, Frei R, Cirilli S, Ferreira V P, Marquillas R A, Barbosa J A, Pereira N S, Belmino I
K C. 2014. High-resolution Hg chemostratigraphy: A contribution to the distinction of chemical fingerprints of the Deccan volcanism and Cretaceous-Paleogene Boundary impact event. Palaeogeogr Palaeoclimatol Palaeoecol, 414: 98-115

Sial A N, Chen J, Lacerda L D, Frei R, Tewari V C, Pandit M K, Gaucher C, Ferreira V P, Cirilli S, Peralta S, Korte C, Barbosa J A, Pereira N S. 2016. Mercury enrichment and $\mathrm{Hg}$ isotopes in Cretaceous-Paleogene boundary successions: Links to volcanism and palaeoenvironmental impacts. Cretac Res, 66: 60-81

Silva M V N, Sial A N, Barbosa J A, Ferreira V P, Neumann V H, De Lacerda L D. 2013. Carbon isotopes, rare-earth elements and mercury geochemistry across the K-T transition of the Paraíba Basin, northeastern Brazil. Geol Soc London Spec Publ, 382: 85104

Sprain C J, Renne P R, Vanderkluysen L, Pande K, Self S, Mittal T. 2019. The eruptive tempo of Deccan volcanism in relation to the Cretaceous-Paleogene boundary. Science, 363: 866-870

Stets J, Ashraf A, Erben H K, Hahn G, Hambach U, Krumsiek K, Thein J, Wurster P. 1996. The Cretaceous-Tertiary boundary in the Nanxiong Basin (continental facies, southeast China). In: MacLeod N, Keller G, eds. Cretaceous-Tertiary Mass Extinctions: Biotic and Environmental Changes. New York: Norton. 349-371

Taylor S R, McLennan S M. 1985. The Continental Crust: Its Composition and Evolution. New York: Oxford. 312

Thibault N, Gardin S. 2010. The calcareous nannofossil response to the end-Cretaceous warm event in the Tropical Pacific. Palaeogeogr Palaeoclimatol Palaeoecol, 291: 239-252

Thibault N, Galbrun B, Gardin S, Minoletti F, Le Callonnec L. 2015. The end-Cretaceous in the southwestern Tethys (Elles, Tunisia): Orbital calibration of paleoenvironmental events before the mass extinction. Int J Earth Sci-Geol Rundsch, 105: 771-795

Thompson R, Oldfield F. 1986. Environmental Magnetism. London: Allen and Unwin. 227

Wan X, Zhao J, Scott R W, Wang P, Feng Z, Huang Q, Xi D. 2013. Late Cretaceous stratigraphy, Songliao Basin, NE China: SK1 cores. Palaeogeogr Palaeoclimatol Palaeoecol, 385: 31-43

Wang C, Feng Z, Zhang L, Huang Y, Cao K, Wang P, Zhao B. 2013. Cretaceous paleogeography and paleoclimate and the setting of SKI borehole sites in Songliao Basin, northeast China. Palaeogeogr Palaeoclimatol Palaeoecol, 385: 17-30

Wang C. 2013. Environmental/climate change in the Cretaceous greenhouse world: Records from Terrestrial scientific drilling of Songliao Basin and adjacent areas of China. Palaeogeogr Palaeoclimatol Palaeoecol, 385: 1-5

Westerhold T, Röhl U, Donner B, McCarren H K, Zachos J C. 2011. A complete high-resolution Paleocene benthic stable isotope record for 
the central Pacific (ODP Site 1209). Paleoceanography, 26: PA2216

Wilf P, Johnson K R, Huber B T. 2003. Correlated terrestrial and marine evidence for global climate changes before mass extinction at the Cretaceous-Paleogene boundary. Proc Natl Acad Sci USA, 100: 599-604

Witt M L I, Mather T A, Pyle D M, Aiuppa A, Bagnato E, Tsanev V I. 2008. Mercury and halogen emissions from Masaya and Telica volcanoes, Nicaragua. J Geophys Res, 113: B06203

Woelders L, Vellekoop J, Kroon D, Smit J, Casadío S, Prámparo M B, Dinarès-Turell J, Peterse F, Sluijs A, Lenaerts J T M, Speijer R P. 2017. Latest Cretaceous climatic and environmental change in the South Atlantic region. Paleoceanography, 32: 466-483

Woelders L, Vellekoop J, Weltje G J, de Nooijer L, Reichart G J, Peterse F, Claeys P, Speijer R P. 2018. Robust multi-proxy data integration, using late Cretaceous paleotemperature records as a case study. Earth Planet Sci Lett, 500: 215-224

Wu H, Zhang S, Jiang G, Huang Q. 2009. The floating astronomical time scale for the terrestrial Late Cretaceous Qingshankou Formation from the Songliao Basin of Northeast China and its stratigraphic and paleoclimate implications. Earth Planet Sci Lett, 278: $308-323$

Yan Y, Xia B, Lin G, Cui X, Hu X, Yan P, Zhang F. 2007. Geochemistry of the sedimentary rocks from the Nanxiong Basin, South China and implications for provenance, paleoenvironment and paleoclimate at the K/T boundary. Sediment Geol, 197: 127140

Zambardi T, Sonke J E, Toutain J P, Sortino F, Shinohara H. 2009. Mercury emissions and stable isotopic compositions at Vulcano Island (Italy). Earth Planet Sci Lett, 277: 236-243

Zhao Z, Mao X, Chai Z, Yang G, Kong P, Ebihara M, Zhao Z. 2002. A possible causal relationship between extinction of dinosaurs and $\mathrm{K} /$ $\mathrm{T}$ iridium enrichment in the Nanxiong Basin, South China: Evidence from dinosaur eggshells. Palaeogeogr Palaeoclimatol Palaeoecol, 178: $1-17$

(责任编委: 朱茂炎) 